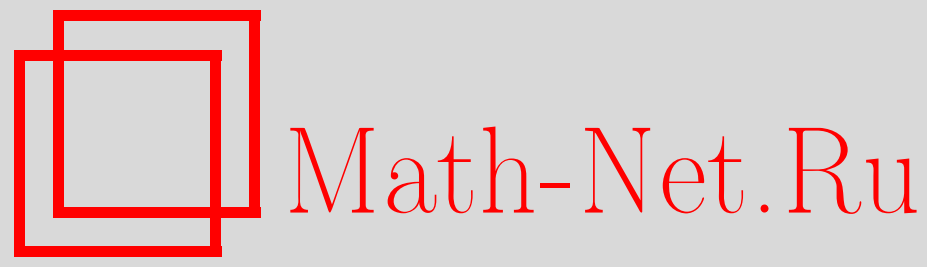

В. Е. Горин, Непересекающиеся пути и ансамбль ортогональных многочленов Хана, Функи. анализ и его прил., 2008, том 42, выпуск 3, 23-44

DOI: https://doi.org/10.4213/faa2910

Использование Общероссийского математического портала MathNet.Ru подразумевает, что вы прочитали и согласны с пользовательским соглашением

http://www . mathnet.ru/rus/agreement

Параметры загрузки:

IP : 54.174 .149 .18

26 апреля 2023 г., 14:12:24

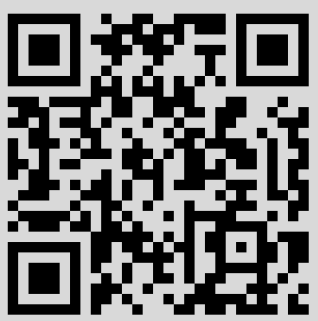


Функиионалъный анализ и его приложения

2008, т. 42, вып. 3, с. 23-44

УДК $519.14+519.21$

\title{
Непересекающиеся пути и ансамбль ортогональных многочленов Хана*
}

\author{
(c) 2008. В. Е. Горин
}

\section{Введение}

В работе рассматривается известная комбинаторная модель детерминантных точечных процессов в размерности $1+1$ (одна пространственная и одна временна́я переменные). Модель зависит от трех натуральных параметров $a, b, c$ и задается равномерным распределением на конечном множестве $\Omega(a, b, c)$ комбинаторных объектов, которое может быть описано несколькими эквивалентными способами: множество трехмерных диаграмм Юнга (иначе говоря, плоских разбиений) в коробке размера $a \times b \times c$; множество замощений ромбами шестиугольника со сторонами $a, b, c, a, b, c$; некоторая модель димеров. См. работы Кона, Ларсена и Проппа [8], Йоханссона [11] и Йоханссона и Норденстама [12]. Соответствия между различными моделями приведены в приложении.

Следуя Йоханссону, используем еще одну интерпретацию, в которой $\Omega(a, b, c)$ отождествляется с некоторым ансамблем непересекающихся ломаных на плоской решетке. Последняя интерпретация и приводит к детерминантным точечным процессам (иначе говоря, случайным точечным конфигурациям), меняющимся со временем. Свойство детерминантности, играющее ключевую роль, означает, что динамические (т. е. пространственно-временные) корреляционные функции модели могут быть получены как миноры некоторой матрицы, называемой динамическим корреляционным ядром. Цель работы - вычисление асимптотики этого ядра в различных предельных режимах.

Если зафиксировать момент времени, то модель определяет случайную конфигурацию точек на одномерной решетке - ортогональный полиномиальный ансамбль Хана. А динамическая модель может быть описана как цепочка таких ансамблей с меняющимися параметрами, ее можно назвать динамическим ансамблем Хана. Динамическое корреляционное ядро есть так называемое расширенное ядро Хана, впервые полученное Йоханссоном в работах [11] и [12]. Основой для вычисления ядра служит теорема Эйнара-Меты [9].

Главный результат работы - вычисление асимптотики расширенного ядра Хана в «bulk limit regime». Ответом является трансляционно-инвариантное ядро $K(x, s ; y, t)$ на $\mathbb{Z}^{2} \times \mathbb{Z}^{2}$, для которого получено простое интегральное представление

$$
K(x, s ; y, t)=\frac{1}{2 \pi i} \oint_{e^{-i \phi}}^{e^{i \phi}}(1+c w)^{t-s} w^{x-y-1} d w .
$$

* Во время написания работы автору оказывали поддержку Фонд поддержки математики им. Леонарда Эйлера, Фонд поддержки молодых ученых «Конкурс Мёбиуса» и РФФИ, грант 07-01-91209. 
Здесь интегрирование ведется по правой стороне единичной окружности при $s \geqslant t$ и по левой в противном случае, а параметры $\phi$ и $c$ зависят от предельного режима (теорема 1).

Статическая версия ядра $(s=t)$ есть хорошо известное дискретное синусядро на $\mathbb{Z} \times \mathbb{Z}[3]$. Полученное динамическое расширение синус-ядра входит в число построенных в [2]. Ядро $K(x, s ; y, t)$ связано простым преобразованием (дуальность частиц и дырок на решетке) с ядром, впервые полученным Окуньковым и Решетихиным [19].

В статье Кениона [13] были исследованы локальные флуктуации в модели, схожей с нашей, в таких же предельных режимах, как и в данной работе. С использованием техники, существенно отличающейся от нашей, было показано, что предельное распределение является одной из так называемых эргодических гиббсовских мер $\mu_{\nu}$ на бесконечном гексагональном графе. Последние были впервые классифицированы в диссертации Шеффилда [21], а в работе [14] были подсчитаны корреляционные ядра для мер $\mu_{\nu}$. В своей статье Кенион указывает, что, по-видимому, его результаты должны быть справедливы в общем случае, однако полное доказательство было приведено только в предположении, что так называемая «предельная форма» («limit shape») не имеет плоских граней. Но как раз в нашем случае предельная форма имеет плоские грани, и, видимо, результат данной работы не является непосредственным следствием результатов работы [13].

Для доказательства теоремы 1 мы не пользуемся приемами работ Кениона, Окунькова и Шеффилда, а, напротив, применяем новый метод.

В литературе имеются тонкие аналитические результаты об асимптотических свойствах дискретных ортогональных полиномиальных ансамблей (см. [1], [11]), но они относятся к статическим, а не динамическим ансамблям. Применить же известный подход - исследовать асимптотику с помощью подходящего интегрального представления ядра - в случае расширенного ядра Хана не получается.

Мы решаем задачу с помощью метода, предложенного в [5]. Хотя в [5] рассматриваются опять-таки лишь статические модели, оказалось, что метод можно адаптировать и к интересующей нас динамической модели. Для вычисления допредельного динамического ядра используются некоторые соотношения, связывающие многочлены Хана с различными параметрами (аналогичный подход применялся в [4]).

Работа построена следующим образом: в $§ 1$ определяется изучаемый случайный процесс и вычисляются его простейшие характеристики; в 2 доказывается детерминантность процесса, а его динамическое корреляционное ядро выражается через ортогональные многочлены Хана; в $§ 3$ формулируется и доказывается основная теорема; наконец, в $§ 4$ анализируются полученные результаты и показывается их связь с теоремами, доказанными в работах [8] и [19].

Автор благодарен А. М. Бородину за постановку задачи, советы и подсказку вида необходимых соотношений для ортогональных многочленов Хана. Автор благодарен также Г. И. Ольшанскому за многочисленные обсуждения и неоценимую помощь в написании данной работы. 


\section{§1. Основные определения и простейшие свойства}

1.1. Базовая модель. Рассмотрим целочисленную решетку $\mathbb{Z}^{2}$ на плоскости $(t, x)$ и два набора точек на ней, $\left\{X_{i}=(0, i-1)\right\}$ и $\left\{Y_{i}=(T, S+i-1)\right\}$, где индекс $i$ меняется от 1 до некоторого фиксированного $N$, а $T$ и $S$ - некоторые фиксированные параметры $(T \geqslant S)$. Мы будем изучать наборы из $N$ непересекающихся путей, проходящих по узлам решетки, при этом $i$-й путь соединяет $X_{i}$ и $Y_{i}$. Пути являются ломаными, у которых каждое звено есть отрезок с левым концом $(t, x) \in \mathbb{Z}^{2}$ и правым концом $(t+1, x)$ или $(t+1, x+1)$. Иными словами, пути состоят из горизонтальных отрезков и отрезков, наклоненных под углом $45^{\circ}$, причем при движении вдоль пути $t$-координата возрастает, а $x$-координата не убывает. Координату $t$ мы часто будем называть временем.

Введем вероятностное пространство всевозможных таких наборов путей и вероятностную меру на нем, считая все наборы равновероятными.

Рассмотрим теперь произвольный момент времени $t$ и набор точек $Z_{1}, \ldots, Z_{N}$ с координатами $\left(t, z_{i}\right)$ соответственно (будем считать, что $z_{i}$ упорядочены по возрастанию). Определена вероятность того, что наш набор путей проходит через точки $Z_{i}$. Эта вероятность естественным образом индуцирует на наборах из $N$ различных натуральных чисел вероятностную меру $P_{t}\left(z_{1}, \ldots, z_{N}\right)$. Далее, изучаемые наборы путей задают случайный процесс с дискретным временем $t=1, \ldots, T$, основным пространством, образованным наборами из $N$ натуральных чисел, и одномерными распределениями $P_{t}$. Обозначим его через $H_{t}$. Haшей задачей будет изучение свойств этого процесса.

Описанный процесс является марковским, это видно из определения марковского процесса как процесса с независимыми прошлым и будущим при фиксированном настоящем.

Обратим внимание, что все пути (соответственно и все конфигурации процесса $H_{t}$ ) расположены внутри шестиугольника, две стороны которого параллельны оси $t$, две другие - оси $x$, а оставшиеся наклонены под углом $45^{\circ}$. Соответствующая иллюстрация приведена на рис. 1.

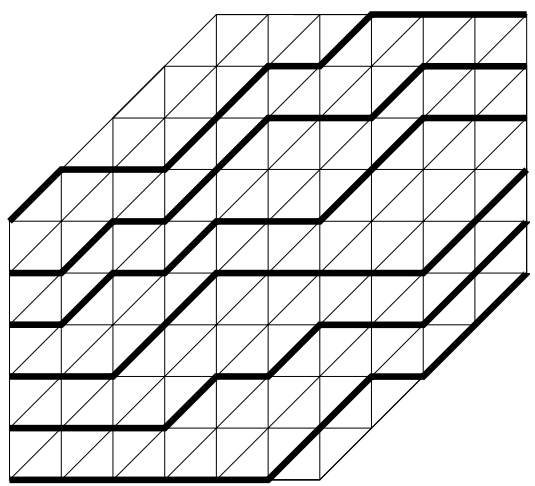

Рис. 1. Набор из шести непересекающихся путей

1.2. Простейшие численные характеристики. Начнем с явного подсчета величины $P_{t}\left(z_{1}, \ldots, z_{n}\right)$

Предложение 1. Количество наборов непересекающихся путей, соединяющих точки $\left(t_{1}, a_{i}\right) u\left(t_{2}, b_{i}\right)$, где $i$ меняется от 1 до $N$, равно $\operatorname{det}_{i, j=1, \ldots, N}\left[\left(\begin{array}{c}t_{2}-t_{1} \\ b_{i}-a_{j}\end{array}\right)\right]$. 
Доказательство. Этот факт является достаточно известным. Прием, используемый для его доказательства, описан в [10].

Из предложения 1 и определений сразу следует, что

$$
P_{t}\left(z_{1}, \ldots, z_{N}\right)=\frac{\operatorname{det}_{i, j=1, \ldots, N}\left[\left(\begin{array}{c}
t \\
z_{i}+1-j
\end{array}\right)\right] \operatorname{det}_{i, j=1, \ldots, N}\left[\left(\begin{array}{c}
T-t \\
S+i-1-z_{j}
\end{array}\right)\right]}{\operatorname{det}_{i, j=1, \ldots, N}\left[\left(\begin{array}{c}
T \\
S+i-j
\end{array}\right)\right]} .
$$

Воспользовавшись теоремой 26 из [16] для подсчета определителей и сделав небольшие упрощения, получаем (здесь и далее мы пользуемся стандартным обозначением $\left.(a)_{i}=a(a+1) \cdots(a+i-1)\right)$, что последнее выражение равно

$$
\begin{aligned}
& \prod_{1 \leqslant i<j \leqslant N}\left(z_{i}-z_{j}\right)^{2} \prod_{i=1}^{N} \frac{1}{z_{i} !\left(t-z_{i}+N-1\right) !\left(S-z_{i}+N-1\right) !\left(T-t-S+z_{i}\right) !} \\
& \times \prod_{i=1}^{N} \frac{(t+1)_{i-1}(T-t+1)_{i-1}(S-i+N) !(T-S+i-1) !}{(T+1)_{i-1}(i-1) !}\left(\frac{t !(T-t) !}{T !}\right)^{N} .
\end{aligned}
$$

После дальнейших преобразований вероятность можно записать в виде

$$
\frac{1}{Z} \prod_{1 \leqslant i<j \leqslant N}\left(z_{i}^{\prime}-z_{j}^{\prime}\right)^{2} \prod_{i=1}^{N}\left(\frac{(\alpha+1)_{z_{i}^{\prime}}(\beta+1)_{M-z_{i}^{\prime}}}{z_{i}^{\prime} !\left(M-z_{i}^{\prime}\right) !}\right),
$$

где $Z$ - нормировочная константа, зависящая от $T, N, S$ и $t$, а координата $z_{i}^{\prime}$ связана с $z_{i}$ простым сдвигом на величину, зависящую от остальных параметров. Все явные формулы, связывающие параметры в различных представлениях весовой функции, будут приведены в следующем параграфе.

Таким образом,

$$
P_{t}\left(z_{1}, \ldots, z_{N}\right)=\frac{1}{Z} \prod_{1 \leqslant i<j \leqslant N}\left(z_{i}^{\prime}-z_{j}^{\prime}\right)^{2} \prod_{i=1}^{N} w_{\alpha, \beta, M}\left(z_{i}^{\prime}\right),
$$

где $w_{\alpha, \beta, M}(x)=\frac{(\alpha+1)_{x}(\beta+1)_{M-x}}{x !(M-x) !}, x=0, \ldots, M$, является весовой функцией для ортогональных многочленов Хана (определение и основные свойства многочленов Хана можно найти в [15]), параметры $\alpha, \beta, M$ зависят от $T, N, S$ и $t$, а $z_{i}^{\prime}$ связана с $z_{i}$ сдвигом начала координат. В дальнейшем мы будем обозначать весовую функцию в момент времени $t$ через $w_{t}(x)$.

\section{§2. Детерминантное представление. Корреляционное ядро}

\section{1. Одномерные распределения.}

Предложение 2. Пусть на множестве целых чисел задан произвольный неотрицательный вес $w(x)$, и пусть $p_{0}, p_{1}, \ldots$ - соответствующие ортогональные многочлены. Далее, пусть на $N$-элементных подмножествах множества челых чисел задана вероятностная мера

$$
P_{t}\left(z_{1}, \ldots, z_{N}\right)=\frac{1}{Z} \prod_{1 \leqslant i<j \leqslant N}\left(z_{i}-z_{j}\right)^{2} \prod_{i=1}^{N} w\left(z_{i}\right) .
$$

Тогда вероятность того, что случайное подмножество содержит заданные 
различные точки $x_{1}<\cdots<x_{k}$, равняется

$$
\underset{i, j=1, \ldots, k}{\operatorname{det}}\left[K\left(x_{i}, x_{j}\right)\right]
$$

где ядро $K$ вычисляется по формуле

$$
K(x, y)=\sqrt{w(x) w(y)} \sum_{n=0}^{N-1} \frac{p_{n}(x) p_{n}(y)}{\left(p_{n}, p_{n}\right)}
$$

Доказательство этой теоремы можно найти в [17]

Такое распределение вероятности на $N$-элементных подмножествах называется ортогональным полиномиальным ансамблем. Легко видеть, что одномерные распределения, возникающие в нашей задаче, имеют именно такой вид, как в последнем предложении.

Теперь явно опишем для рассматриваемого процесса $H_{t}$ одномерные распределения и используемые ортогональные многочлены и весовые функции.

Итак, в нашей модели весовая функция записывается в виде

$$
\begin{aligned}
w_{t}(x) & =\frac{c}{z_{i} !(t-x+N-1) !(S-x+N-1) !(T-t-S+x) !} \\
& =\frac{(\alpha+1)_{x^{\prime}}(\beta+1)_{M-x^{\prime}}}{x^{\prime} !\left(M-x^{\prime}\right) !}
\end{aligned}
$$

(здесь $c$ - некоторая константа, не зависящая от $x$, а $x^{\prime}$ отличается от $x$ некоторым сдвигом). Обратим внимание на то, что носитель весовой функции меняется со временем, а именно, при росте $t$ на единицу носитель либо не меняется, либо к нему добавляется одна точка сверху, либо из него удаляется одна точка снизу, либо происходит одновременно и добавление, и удаление (см. рис. 1). Обозначим множество точек в носителе функции $w_{t}$ через $\mathfrak{X}_{t}$.

Обозначим через $Q_{k}\left(x^{\prime}, \alpha, \beta, M\right)$ ортогональный многочлен Хана степени $k$ с параметрами $\alpha, \beta, M$. В соответствии с [15] такие многочлены определены при $x^{\prime}=0, \ldots, M, k=0, \ldots, M$ и ортогональны относительно веса

$$
\frac{(\alpha+1)_{x^{\prime}}(\beta+1)_{M-x^{\prime}}}{x^{\prime} !\left(M-x^{\prime}\right) !}
$$

Многочлены Хана можно выразить через обобщенную гипергеометрическую функцию:

$$
Q_{k}\left(x^{\prime}, \alpha, \beta, M\right)={ }_{3} F_{2}\left(\begin{array}{c}
-k,-x^{\prime}, k+\alpha+\beta+1 \\
-M, \alpha+1
\end{array}\right)(1) .
$$

Обозначим через $H_{k}^{t}(x)$ многочлен Хана $Q_{k}$, отвечающий моменту времени $t$ и сдвинутый так, чтобы его область определения совпадала с $\mathfrak{X}_{t}$.

В зависимости от соотношения между $t$ и остальными параметрами возникают 4 случая, в которых $\alpha, \beta, M, x^{\prime}$ по-разному выражаются через параметры 
задачи:

(I) $t<S+1, t<T-S+1$ :

$M=t+N-1, \alpha=-S-N, \beta=S-T-N, x^{\prime}=x$,

$H_{t}^{k}(x)=Q_{k}\left(x^{\prime}, \alpha, \beta, M\right)={ }_{3} F_{2}\left(\begin{array}{c}-k,-x^{\prime}, k+\alpha+\beta+1 \\ -M, \alpha+1\end{array}\right)(1)={ }_{3} F_{2}\left(\begin{array}{l}-k,-x, k-2 N-T+1 \\ -S-N+1,-t-N+1\end{array}\right)(1)$,

$0 \leqslant x \leqslant M=t+N-1,0 \leqslant k \leqslant M=t+N-1$;

(II) $S-1<t<T-S+1$ :

$M=S+N-1, \alpha=-t-N, \beta=t-N-T, x^{\prime}=x$,

$H_{t}^{k}(x)=Q_{k}\left(x^{\prime}, \alpha, \beta, M\right)={ }_{3} F_{2}\left(\begin{array}{c}-k,-x^{\prime}, k+\alpha+\beta+1 \\ -M, \alpha+1\end{array}\right)(1)={ }_{3} F_{2}\left(\begin{array}{l}-k,-x, k-2 N-T+1 \\ -S-N+1,-t-N+1\end{array}\right)(1)$,

$0 \leqslant x \leqslant M=s+N-1, \leqslant \leqslant M=s+N-1$;

(III) $T-S-1<t<S+1$ :

$M=t+N-S-1, \alpha=-T+t-N, \beta=-t-N, x^{\prime}=T-t-s+x$,

$H_{t}^{k}(x)=Q_{k}\left(x^{\prime}, \alpha, \beta, M\right)={ }_{3} F_{2}\left(\begin{array}{c}-k,-x+t+s-T, k-2 N-T+1 \\ -T+t-N+1,-T+S-N+1\end{array}\right)(1)$,

$-T+t+S \leqslant x^{\prime} \leqslant t+N-1,0 \leqslant k \leqslant T+N-S-1$;

(IV) $t>T-S-1, t>S-1$ :

$M=T-t+N-1, \alpha=-T-N+S, \beta=-S-N, x^{\prime}=T-t-s+x$,

$H_{t}^{k}(x)=Q_{k}\left(x^{\prime}, \alpha, \beta, M\right)={ }_{3} F_{2}\left(\begin{array}{c}-k,-x+t+s-T, k-2 N-T+1 \\ -T+t-N+1,-T+S-N+1\end{array}\right)(1)$,

$-T+t+S \leqslant x^{\prime} \leqslant S+N-1,0 \leqslant k \leqslant T+N-t-1$.

Заметим, что в случаях (I) и (II) выражения многочленов $H_{k}^{t}$ через гипергеометрическую функцию совпадают. Такая же ситуация и в случаях (III) и (IV).

Введем еще одно обозначение:

$$
f_{n}^{t}(x)=\frac{H_{n}^{t}(x) \sqrt{w_{t}(x)}}{\sqrt{\left(H_{n}^{t}, H_{n}^{t}\right)}} .
$$

Здесь $\left(H_{n}^{t}, H_{n}^{t}\right)$ - квадрат нормы многочлена $H_{n}^{t}$ относительно весовой функции $w_{t}(x)$. В соответствии с [15]

$$
\begin{aligned}
\left(H_{n}^{t}, H_{n}^{t}\right) & =\left(Q_{k}\left(x^{\prime}, \alpha, \beta, M\right), Q_{k}\left(x^{\prime}, \alpha, \beta, M\right)\right) \\
& =\frac{(-1)^{k}(k+\alpha+\beta+1)_{N+1}(\beta+1)_{k} k !}{(2 k+\alpha+\beta+1)(\alpha+1)_{k}(-N)_{k} N !} .
\end{aligned}
$$

Функции $f_{n}^{t}(x)$ образуют ортонормированный базис в пространстве $l_{2}\left(\mathfrak{X}_{t}\right)$.

Теперь ядро $K$ из предложения 2 можно для нашей задачи записать в виде

$$
K(x, y)=\sum_{n=0}^{N-1} f_{n}^{t}(x) f_{n}^{t}(y) .
$$

2.2. Динамическая детерминантность. Будем называть детерминантным любой случайный процесс $X(t)$ со значениями в подмножествах некоторого 
счетного множества, для которого существует функция $K(x, s ; y, t)$, такая, что для любого набора различных пар $\left(x_{1}, t_{1}\right), \ldots,\left(x_{n}, t_{n}\right)$

$$
\operatorname{Prob}\left\{x_{1} \in X\left(t_{1}\right), x_{2} \in X\left(t_{2}\right), \ldots, x_{n} \in X\left(t_{n}\right)\right\}=\operatorname{det}_{i, j=1, \ldots, n}\left[K\left(x_{i}, t_{i} ; x_{j}, t_{j}\right)\right] .
$$

Функцию $K$ будем называть динамическим корреляиионным ядром, а сами вероятности - динамическими корреляционными функциями.

Для доказательства детерминантности процесса $H_{t}$ нам понадобится общее абстрактное утверждение.

Предложение 3. Пусть для каждого момента времени $t$ заданы ортонормированная система $\left\{f_{n}^{t}\right\}$ в $l_{2}(\{0,1, \ldots, L\})$ (она может образовъвать базис не всего пространства, а лишь $l_{2}\left(L_{t}\right)$, где $\left.L_{t} \subset\{0,1, \ldots, L\}\right)$ и набор чисел $c_{0}^{t}, c_{1}^{t}, \ldots$ ППоложсим

$$
v_{t, t+1}(x, y)=\sum_{n \geqslant 0} c_{n}^{t} f_{n}^{t}(x) f_{n}^{t+1}(y) .
$$

Кроме того, пусть имеется марковский процесс $X_{t}$ с дискретным временем $t$, принимающий значения в $N$-элементных подмножествах множества $\{0,1, \ldots, L\}$, с одномерными распределениями

$$
\left(\underset{i, j=1, \ldots, N}{\operatorname{det}}\left[f_{i-1}^{t}\left(x_{j}\right)\right]\right)^{2}
$$

и переходными вероятностями

$$
\frac{\operatorname{det}\left[v_{t, t+1}\left(x_{i}, y_{j}\right)\right] \operatorname{det}\left[f_{i-1}^{t+1}\left(y_{j}\right)\right]}{\operatorname{det}\left[f_{i-1}^{t}\left(x_{j}\right)\right] \prod_{n=0}^{N-1} c_{n}^{t}} .
$$

Toгдa

$p_{n}\left(x_{1}, k_{1} ; \ldots ; x_{n}, k_{n}\right):=\operatorname{Prob}\left\{x_{1} \in X_{k_{1}}, \ldots, x_{n} \in X_{k_{n}}\right\}=\operatorname{det}_{i, j=1, \ldots, n}\left[K\left(x_{i}, k_{i} ; x_{j}, k_{j}\right)\right]$,

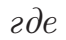

$$
\begin{aligned}
& K(x, k ; y, l)=\sum_{i=0}^{N-1} \frac{1}{c_{i}^{l, k}} f_{i}^{k}(x) f_{i}^{l}(y), \quad k \geqslant l, \\
& K(x, k ; y, l)=-\sum_{i \geqslant N} c_{i}^{k, l} f_{i}^{k}(x) f_{i}^{l}(y), \quad k<l, \\
& c_{i}^{k, k}=1, \quad c_{i}^{k, l}=c_{i}^{k} c_{i}^{k+1} \cdots c_{i}^{l-1} .
\end{aligned}
$$

Это предложение является переформулировкой теоремы, доказанной Эйнаром и Метой в [9]. Другая схожая его формулировка использовалась в [4].

Стоит обратить внимание на то, что динамическое корреляционное ядро не является канонически определенным объектом. Инвариантами процесса являются лишь корреляционные функции, которые не меняются при преобразованиях динамического корреляционного ядра вида

$$
K^{*}(x, s ; y, t)=K(x, s ; y, t) \frac{F(x, s)}{F(y, t)},
$$

где $F$ - произвольная функция. Легко видеть, что в результате такого преобразования значения определителей не меняются. 
Лемма 4. Переходные вероятности процесса $H_{t}$ за один временной шаг вычисляются по формуле

$$
\begin{gathered}
P_{t, t+1}\left(x_{1}, \ldots, x_{N} ; y_{1}, \ldots, y_{N}\right):=\operatorname{Prob}\left\{H_{t+1}=\left(y_{1}, \ldots, y_{N}\right) \mid H_{t}=\left(x_{1}, \ldots, x_{N}\right)\right\} \\
=\frac{\prod_{i<j}\left(y_{j}-y_{i}\right) \prod_{i: y_{i}=x_{i}+1}\left(N+S-x_{i}-1\right) \prod_{i: y_{i}=x_{i}}\left(x_{i}+T-t-S\right)}{(T-t)_{N} \cdot \prod_{i<j}\left(x_{j}-x_{i}\right)}
\end{gathered}
$$

если каждая из разностей $y_{i}-x_{i}$ равна нулю или единице, и равны нулю в противном случае.

Справедливость последней формулы проверяется непосредственно по определению с использованием предложения 1 и формул для подсчета определителей, описанных в разд. 1.2.

Предложение 5. Процесс $H_{t}$ удовлетворяет условиям предложения 3 и, таким образом, является детерминантным.

Здесь ортонормированной системой из формулировки предложения 3 является система функций $f_{n}^{t}$, определенных в разд. 2.1 , а коэффициенты $c_{i}^{t}$ вычисляются по формуле

$$
c_{i}^{t}=\sqrt{\left(1-\frac{i}{t+N}\right)\left(1-\frac{i}{T+N-t-1}\right)} .
$$

Доказательство предложения 5. Нам понадобятся три леммы.

Лемма 6. Для ортогональных многочленов Хана имеют место следующие два соотношения:

$$
\begin{array}{r}
x Q_{k}(x-1 ; \alpha, \beta, M-1)+(M-x) Q_{k}(x ; \alpha, \beta, M-1)=M Q_{k}(x ; \alpha, \beta, M), \\
x Q_{k}(x-1 ; \alpha+1, \beta-1, M)+(-x-\alpha-1) Q_{k}(x ; \alpha+1, \beta-1, M) \\
=-(\alpha+1) Q_{k}(x ; \alpha, \beta, M),
\end{array}
$$

которые эквивалентны одному соотношению для функиии ${ }_{3} F_{2}$ :

$x \cdot{ }_{3} F_{2}\left(\begin{array}{c}-k, a,-x+1 \\ b, c+1\end{array}\right)(1)-(c+x) \cdot{ }_{3} F_{2}\left(\begin{array}{c}-k, a,-x \\ b, c+1\end{array}\right)(1)=-c \cdot{ }_{3} F_{2}\left(\begin{array}{c}-k, a,-x \\ b, c\end{array}\right)(1)$.

Доказательство. Справедлива следующая формула (см. [15]):

$Q_{k}(x, \alpha, \beta, M)={ }_{3} F_{2}\left(\begin{array}{c}-k,-x, k+\alpha+\beta+1 \\ -M, \alpha+1\end{array}\right)(1)=\sum_{i=0}^{k} \frac{(-k)_{i}(-x)_{i}(k+\alpha+\beta+1)_{i}}{(-M)_{i}(\alpha+1)_{i} i !}$.

Из нее вытекает, что

$$
\begin{aligned}
& x Q_{k}(x-1 ; \alpha, \beta, M-1)+(M-x) Q_{k}(x ; \alpha, \beta, M-1)-M Q_{k}(x ; \alpha, \beta, M) \\
& \quad=\sum_{i=0}^{k} \frac{(-k)_{i}(k+\alpha+\beta+1)_{i}}{(\alpha+1)_{i} i !}\left[x \frac{(-x+1)_{i}}{(-M+1)_{i}}+(M-x) \frac{(-x)_{i}}{(-M+1)_{i}}-M \frac{(-x)_{i}}{(-M)_{i}}\right] \\
& =\sum_{i=0}^{k} \frac{(-k)_{i}(-x)_{i}(k+\alpha+\beta+1)_{i}}{(-M+1)_{i}(\alpha+1)_{i} i !}[(x-k)+(M-x)-(M-k)]=0 .
\end{aligned}
$$

Легко видеть, что второе соотношение эквивалентно уже доказанному. 
Лемма 7. Имеет место равенство

$$
\begin{gathered}
\sum_{k=0}^{M} \frac{(2 k+\alpha+\beta+1)(\alpha+1)_{k}(-M)_{k} M !}{(-1)^{k}(k+\alpha+\beta+1)_{M+1}(\beta+1)_{k} k !} Q_{k}(x, \alpha, \beta, M) Q_{k}(y, \alpha, \beta, M) \\
=\delta_{x, y} / \frac{(\alpha+1)_{x}(\beta+1)_{M-x}}{x !(M-x) !} .
\end{gathered}
$$

Эта формула носит название «двойственное соотношение ортогональности». Ее можно найти в [15].

Лемма 8. Имеет место равенство

$$
\begin{aligned}
\sqrt{\frac{w_{t+1}(y)}{w_{t}(x)}} \sum_{k \geqslant 0} c_{k}^{t} f_{k}^{t}(x) f_{k}^{t+1}(y) & \\
& =\frac{S+N-1-x}{\sqrt{(t+N)(T+N-t-1)}} \delta_{x+1}^{y}+\frac{T-t-S+x}{\sqrt{(t+N)(T+N-t-1)}} \delta_{x}^{y} .
\end{aligned}
$$

Здесъ $c_{i}^{t} u f_{k}^{t}(x)$ - это коэффициенты и функиии, определеннъе после формулировки предложения 5, a $w_{t}(x)$ - весовая функция многочленов Хана.

Доказательство. Для доказательства достаточно вспомнить определение функций $f_{k}^{t}(x)$, подставить значения весовой функции и всех параметров, затем в случаях (I), (II) выразить $f_{k}^{t+1}(y)$ через $f_{k}^{t}(y)$ и $f_{k}^{t}(y-1)$ с помощью соотношения $(*)$ и применить последнюю лемму. В случаях (III) и (IV) надо выразить $f_{k}^{t}(x)$ через $f_{k}^{t+1}(x)$ и $f_{k}^{(t+1)}(x+1)$, пользуясь соотношением $(* *)$, и снова применить двойственное соотношение ортогональности.

Вернемся к доказываемому предложению.

Тот факт, что одномерные распределения представляются в нужном для применения предложения 3 виде, следует из предложения 2 , в котором полагаем $k$ равным $N$ (в этом случае само предложение 2 почти очевидно), и того, что

$$
\begin{aligned}
\left(\underset{i, j=1, \ldots, N}{\operatorname{det}}\left[f_{i-1}^{t}\left(x_{j}\right)\right]\right)^{2} & =\operatorname{det}_{i, j=1, \ldots, N}\left[f_{i-1}^{t}\left(x_{j}\right)\right] \cdot \operatorname{det}_{i, j=1, \ldots, N}\left[f_{j-1}^{t}\left(x_{i}\right)\right] \\
& =\operatorname{det}\left(\left[f_{i-1}^{t}\left(x_{j}\right)\right] \cdot\left[f_{j-1}^{t}\left(x_{i}\right)\right]\right)=\operatorname{det}_{i, j=1, \ldots, N}\left[K\left(x_{j}, x_{i}\right)\right]
\end{aligned}
$$

(здесь $K(x, y)$ - это ядро, определенное в предложении 2).

Остается показать, что переходные вероятности также имеют нужный вид, т. e.

$$
P_{t, t+1}\left(x_{1}, \ldots, x_{N} ; y_{1}, \ldots, y_{N}\right)=\frac{\operatorname{det}\left[v_{t, t+1}\left(x_{i}, y_{j}\right)\right] \operatorname{det}\left[f_{i-1}^{t+1}\left(y_{j}\right)\right]}{\operatorname{det}\left[f_{i-1}^{t}\left(x_{j}\right)\right] \prod_{n=0}^{N-1} c_{n}^{t}}
$$

(все матрицы, от которых берутся определители, имеют размер $N \times N$, и мы не будем в дальнейшем его явно указывать). Здесь

$$
v_{t, t+1}(x, y)=\sum_{k \geqslant 0} c_{k}^{t} f_{k}^{t}(x) f_{k}^{t+1}(y) .
$$


По уже доказанному

$$
\begin{aligned}
v_{t, t+1}(x, y) & =\sqrt{\frac{w_{t}(x)}{w_{t+1}(y)}}\left[\frac{S+N-1-x}{\sqrt{(t+N)(T+N-t-1)}} \delta_{x+1}^{y}+\frac{T-t-S+x}{\sqrt{(t+N)(T+N-t-1)}} \delta_{x}^{y}\right], \\
& \operatorname{det}\left[f_{i-1}^{t+1}\left(y_{j}\right)\right]=\sqrt{P_{t+1}\left(y_{1}, \ldots, y_{N}\right)}, \quad \operatorname{det}\left[f_{i-1}^{t}\left(x_{j}\right)\right]=\sqrt{P_{t}\left(x_{1}, \ldots, x_{N}\right)} .
\end{aligned}
$$

Подставляя выражения для весовой функции и вероятности $N$-элементного набора, получаем

$$
\begin{aligned}
& \frac{\operatorname{det}\left[v_{t, t+1}\left(x_{i}, y_{j}\right)\right] \operatorname{det}\left[f_{i-1}^{t+1}\left(y_{j}\right)\right]}{\operatorname{det}\left[f_{i-1}^{t}\left(x_{j}\right)\right] \prod_{i=0}^{N-1} c_{i}^{t}} \\
& \quad=\frac{\prod_{i=1}^{N} \sqrt{\frac{w_{t+1}\left(y_{i}\right)}{w_{t}\left(x_{i}\right)} \frac{(t+i)(T-t)(t+1)^{N}}{(t+1)(T-t+i-1)(T-t)^{N}}} \operatorname{det}\left[v_{t, t+1}\left(x_{i}, y_{j}\right)\right]}{\prod_{i=0}^{N-1} c_{i}^{t}} \prod_{i<j} \frac{y_{j}-y_{i}}{x_{j}-x_{i}} \\
& =\frac{\operatorname{det}\left[(S+N-x-1) \delta_{x_{i}+1}^{y_{j}}+(T-t-S+x) \delta_{x_{i}}^{y_{j}}\right]}{(T-t)_{N}} \prod_{i<j} \frac{y_{j}-y_{i}}{x_{j}-x_{i}} .
\end{aligned}
$$

Будем считать, что $x_{i}$ и $y_{i}$ упорядочены в порядке возрастания.

Имеется два случая: либо каждая из разностей $y_{i}-x_{i}$ принимает значение 0 или 1 , либо это не так.

В последнем случае матрица под знаком определителя в последней строке формулы $(* * *)$ имеет нулевую строчку или столбец; следовательно, определитель равен нулю, но и переходная вероятность тоже нулевая.

В первом случае эта матрица под знаком определителя является блочнодиагональной, причем нули в ней расположены так, что каждый блок есть либо нижнетреугольная, либо верхнетреугольная матрица. Мы можем заключить, что определитель матрицы равен произведению ее диагональных элементов и значение выражения $(* * *)$ совпадает с переходной вероятностью для процесса $H_{t}$, подсчитанной в лемме 4.

Таким образом, процесс подпадает под условия предложения 3, и мы можем заключить, что он детерминантен.

\section{§3. Переход к пределу в корреляционных ядрах: операторный метод}

3.1. Формулировка результата. Нас интересует предельное распределение (предельное корреляционное ядро) в следующем предельном режиме.

Зафиксируем числа $\widetilde{S}, \widetilde{T}, \widetilde{N}, \tilde{t}, \tilde{x}$. Пусть вспомогательный параметр $\rho$ стремится к $\infty$ и

$$
\begin{gathered}
S=\rho \widetilde{S}+o(\rho), \quad T=\rho \widetilde{T}+o(\rho), \quad N=\rho \tilde{N}+o(\rho), \quad t=\rho \tilde{t}+\hat{t}, \\
s=\rho \tilde{t}+\hat{s}, \quad x=\rho \tilde{x}+\hat{x}, \quad y=\rho \tilde{x}+\hat{y} .
\end{gathered}
$$

Такой предел можно интерпретировать следующим образом: мы фиксируем пропорции $\widetilde{T}, \widetilde{S}, \widetilde{N}$ шестиугольника, внутри которого расположены пути, и точку $(\tilde{t}, \tilde{x})$ внутри него. Теперь мы начинаем раздувать шестиугольник с помощью гомотетии с коэффициентом $\rho$ и центром в точке $(\tilde{t}, \tilde{x})$. Таким образом, 
количество путей начинает возрастать, и мы изучаем получающуюся картинку вблизи точки $(\tilde{t}, \tilde{x})$.

Напомним формулу для корреляционного ядра, в которой мы хотим перейти к пределу:

$$
K(x, s ; y, t)= \begin{cases}-\sum_{i \geqslant N}\left(\prod_{j=s}^{t-1} c_{i}^{j}\right) f_{i}^{s}(x) f_{i}^{t}(y), \quad s<t, \\ \sum_{i=0}^{N-1}\left(\prod_{j=t}^{s-1} \frac{1}{c_{i}^{j}}\right) f_{i}^{s}(x) f_{i}^{t}(y), \quad s \geqslant t .\end{cases}
$$

Здесь $f_{i}^{s}(x)$ и $c_{i}^{j}-$ функции и коэффициенты, определенные в $\S 2$.

Теорема 1. Пусть в исходной модели все параметры $S, T, N, t, s, x, y$ стремятся $к$ бесконечности так, что

$$
\begin{gathered}
S=\rho \widetilde{S}+o(\rho), \quad T=\rho \tilde{T}+o(\rho), \quad N=\rho \tilde{N}+o(\rho), \quad t=\rho \tilde{t}+\hat{t}, \\
s=\rho \tilde{t}+\hat{s}, \quad x=\rho \tilde{x}+\hat{x}, \quad y=\rho \tilde{x}+\hat{y}
\end{gathered}
$$

где вспомогательный параметр $\rho$ стремится $\kappa \infty$. Тогда существует предельный точечный процесс на $\mathbb{Z}^{2}$. Он является детерминантным и имеет трансляиионно-инвариантное корреляционное ядро

$$
K(\hat{x}, \hat{s} ; \hat{y}, \hat{t})=\frac{1}{2 \pi i} \oint_{e^{-i \phi}}^{e^{i \phi}}(1+c w)^{\hat{t}-\hat{s}} w^{\hat{x}-\hat{y}-1} d w .
$$

Здесь интегрирование ведется по правой стороне единичной окружсности при $\hat{s} \geqslant \hat{t}$ и по левой в противном случае. Параметр с имеет вид

$$
c=\sqrt{\frac{\tilde{x}(\widetilde{S}+\widetilde{N}-\tilde{x})}{(\widetilde{T}-\tilde{t}-\widetilde{S}+\tilde{x})(\tilde{t}+\widetilde{N}-\tilde{x})}},
$$

а угол ф вычисляется по формуле

$$
\phi=\arccos \frac{-\tilde{N}(\tilde{N}+\widetilde{T})+(-\tilde{x}+\widetilde{S}+\tilde{N})(\tilde{t}+\tilde{N}-\tilde{x})+\tilde{x}(\widetilde{T}+\tilde{x}-\widetilde{S}-\tilde{t})}{2 \sqrt{\tilde{x}(-\tilde{x}+\widetilde{S}+\tilde{N})(\tilde{t}+\tilde{N}-\tilde{x})(\tilde{x}+\widetilde{T}-\widetilde{S}-\tilde{t})}} .
$$

Если выражение под знаком арккосинуса больше 1 , то полагаем $\phi=0$, а если меньше -1 , то $\phi=\pi$.

Существование процесса, задаваемого предельными корреляционными функциями, легко следует из сходимости этих функций. Доказательство этого простого факта можно найти, например, в [2, лемма 4.1].

Доказательство сходимости корреляционных ядер проведем в два этапа.

3.2. Статический случай. Пусть сначала $\hat{s}-\hat{t}=0$, т. е. мы изучаем лишь «статическое» распределение в некоторый фиксированный момент времени.

Воспользуемся методом, предложенным А. М. Бородиным и Г. И. Ольшанским в статье [5].

Продолжим функции $f_{i}^{t}(x)$, принадлежащие пространству $l_{2}\left(\mathfrak{X}_{t}\right)$, до функций на $\mathbb{Z}$, доопределив их нулями. Теперь мы можем считать, что все рассматриваемые функции $f_{i}^{t}$ принадлежат одному и тому же пространству $l_{2}(\mathbb{Z})$. 
Имеем

$$
K_{t}(x, y)=\sum_{i=0}^{N-1} f_{i}^{t}(x) f_{i}^{t}(y) .
$$

Заметим, что $K_{t}(x, y)$ является матричным элементом для оператора проектирования на линейную оболочку первых $N$ ортогональных функций $f_{i}^{t}, i=$ $0, \ldots, N-1$, в пространстве $l_{2}(\mathbb{Z})$.

Оказывается, что проще искать не предел матричных элементов, а предел всего оператора. Для этого обратим внимание на то, что функции $f_{n}^{t}(x)$, получаемые из ортогональных многочленов Хана, являются собственными векторами некоторого разностного оператора (он явно указан ниже). Оператор проектирования можно представить как спектральный проектор на интервал, содержащий первые $N$ собственных значений этого разностного оператора. Теперь для нахождения предела спектральных проекторов перейдем к пределу в самих разностных операторах. Обратим внимание на то, что одновременно меняется и спектральный интервал, и разностный оператор. Для обоснования корректности предельного перехода воспользуемся несколькими теоремами из функционального анализа.

Возьмем за общую область существенной самосопряженности всех рассматриваемых разностных операторов множество $l_{2}^{0}(\mathbb{Z})$ финитных векторов из $l_{2}(\mathbb{Z})$, т. е. конечных линейных комбинаций базисных векторов. Как будет видно из дальнейшего, рассматриваемые разностные операторы сильно сходятся на этой области. По теореме VIII.25 из [20] из этого следует сходимость в сильном резольвентном смысле. А из этой последней, непрерывности спектра предельного оператора и теоремы VIII.24 из [20] можно заключить, что рассматриваемые спектральные проекторы, ассоциированные с разностными операторами, сильно сходятся на множестве финитных векторов к предельному спектральному проектору, ассоциированному с предельным разностным оператором.

Проведем теперь само вычисление. $[15])$ :

Для многочленов Хана имеет место следующее разностное соотношение (см.

$$
\begin{aligned}
k(k+\alpha+\beta+1) & Q_{k}\left(x^{\prime} ; \alpha, \beta, M\right)=\left(x^{\prime}+\alpha+1\right)(M-x) Q_{k}\left(x^{\prime}-1 ; \alpha, \beta, M\right) \\
+ & \left(\left(x^{\prime}+\alpha+1\right)(M-x)+x^{\prime}\left(x^{\prime}-\beta-M-1\right)\right) Q_{k}\left(x^{\prime} ; \alpha, \beta, M\right) \\
+ & x^{\prime}\left(x^{\prime}-\beta-M-1\right) Q_{k}\left(x^{\prime}+1 ; \alpha, \beta, M\right) .
\end{aligned}
$$

Перепишем это соотношение в терминах ортонормированных функций $f_{k}^{t}\left(x^{\prime}\right)$; подчеркнем, что здесь используется переменная $x^{\prime}$, а не $x$ (как было показано в разд. 2.1, в случаях (I), (II) $x=x^{\prime}$, а в (III) и (IV) $\left.x \neq x^{\prime}\right)$ :

$$
\begin{aligned}
& \frac{k(k+\alpha+\beta+1)}{\rho^{2}} f_{k}^{t}\left(x^{\prime}\right)=\frac{\sqrt{\left(x^{\prime}+1\right)\left(M-x^{\prime}\right)\left(\beta+M-x^{\prime}\right)\left(\alpha+x^{\prime}\right)}}{\rho^{2}} f_{k}^{t}\left(x^{\prime}+1\right) \\
&-\frac{\left(x^{\prime}+\alpha+1\right)\left(x^{\prime}-M\right)+^{\prime} x\left(x^{\prime}-\beta-M-1\right)}{\rho^{2}} f_{k}^{t}\left(x^{\prime}\right) \\
&+\frac{\sqrt{x^{\prime}\left(M-x^{\prime}+1\right)\left(\beta+M-x^{\prime}\right)\left(\alpha+x^{\prime}\right)}}{\rho^{2}} f_{k}^{t}\left(x^{\prime}-1\right) .
\end{aligned}
$$

Мы поделили соотношения на $\rho^{2}$ для того, чтобы пределы, изучаемые в дальнейшем, существовали. 
Пусть теперь $H$ - разностный оператор, задаваемый правой частью последнего соотношения при тех $x$, при которых определены многочлены Хана, и, например, тождественный оператор при остальных $x$. Матрица оператора $H$ имеет вид

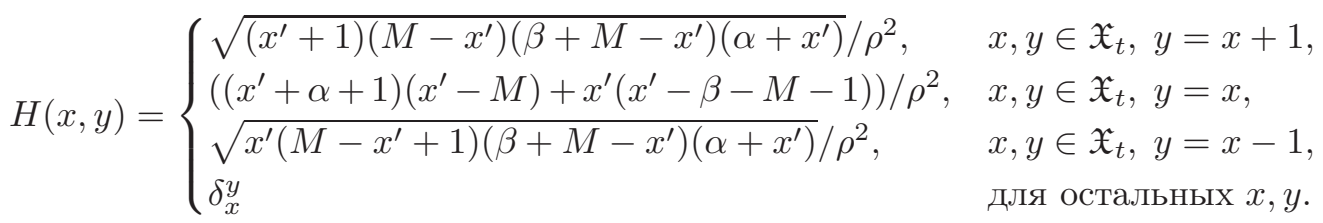

Ясно, что $H$ - самосопряженный оператор.

Нас интересует проектор на собственные векторы, отвечающие части спектра оператора при $k \in\{0, \ldots, N-1\}$, т. е. собственным значениям

$$
k(k+\alpha+\beta+1) / \rho^{2}, \quad k \in\{0, \ldots, N-1\} .
$$

Заметим, что полный спектр оператора $H$ состоит из отрицательных значений, получаемых по последней формуле при $k$, пробегающем все значения от 0 до $M$, и единицы, возникающей из-за формального продолжения оператора. В нашей модели $\alpha$ и $\beta$ - достаточно большие по модулю отрицательные числа и функция $k(k+\alpha+\beta+1)$ отрицательна и монотонна при допустимых значениях $k$. Следовательно, мы можем заменить проектор на собственные пространства, связанные с дискретным набором точек спектра, проектором, ассоциированным с содержащим их интервалом, т. е. искомый проектор $P$ - спектральный проектор, отвечающий отрезку

$$
\left[(N-1)(N+\alpha+\beta) / \rho^{2}, 0\right] .
$$

Подставим значения $\alpha, \beta$ и прочих параметров и выразим оператор $H$ и проектор $P$ через $\rho$ и параметры $\widetilde{S}, \widetilde{T}, \widetilde{N}, \tilde{t}, \tilde{x}$ :

$$
S=\rho \widetilde{S}+o(\rho), \quad T=\rho \widetilde{T}+o(\rho), \quad N=\rho \tilde{N}+o(\rho), \quad t=\rho \tilde{t}+\hat{t}, \quad x=\rho \tilde{x}+\hat{x} .
$$

Получим семейство операторов $P_{\rho}$ в пространстве $l_{2}(\mathbb{Z})$, где аргументом является $\hat{x}$. Необходимо найти

$$
\widehat{P}=\lim _{\rho \rightarrow \infty} P_{\rho}
$$

Для применения теоремы VII.24 из [20] необходимо, чтобы семейство разностных операторов $H_{\rho}$, полученных подстановкой параметров предельного режима, сходилось к предельному $\widehat{H}$ в смысле сильной операторной сходимости на множестве всех финитных векторов, а такая сходимость будет следовать из сходимости матричных элементов, так как в нашем случае все операторы $H_{\rho}$ задаются трехдиагональными матрицами.

Переходя к пределу в матричных элементах, получаем, что $\widehat{H}-$ симметрический оператор с трехдиагональной матрицей, у которой на главной диагонали стоит число

$$
\begin{aligned}
A & =\lim _{\rho \rightarrow \infty}\left(-\frac{\left(x^{\prime}+\alpha+1\right)\left(x^{\prime}-M\right)+x^{\prime}\left(x^{\prime}-\beta-M-1\right)}{\rho^{2}}\right) \\
& =-(\widetilde{S}+\tilde{N}-\tilde{x})(\tilde{t}+\tilde{N}-\tilde{x})-\tilde{x}(\tilde{x}+\widetilde{T}-\widetilde{S}-\tilde{t}),
\end{aligned}
$$


а над и под главной диагональю стоит число

$$
\begin{aligned}
B & =\lim _{\rho \rightarrow \infty} \frac{\sqrt{\left(x^{\prime}+1\right)\left(M-x^{\prime}\right)\left(\beta+M-x^{\prime}\right)\left(\alpha+x^{\prime}\right)}}{\rho^{2}} \\
& =\lim _{\rho \rightarrow \infty} \frac{\sqrt{\left(x^{\prime}\right)\left(M-x^{\prime}+1\right)\left(\beta+M-x^{\prime}\right)\left(\alpha+x^{\prime}\right)}}{\rho^{2}} \\
& =\sqrt{(\widetilde{S}+\tilde{N}-\tilde{x})(\tilde{t}+\tilde{N}-\tilde{x}) \tilde{x}(\tilde{x}+\tilde{T}-\tilde{S}-\tilde{t})} .
\end{aligned}
$$

Обратим внимание на то, что, хотя параметры $\alpha, \beta, M$ и $x^{\prime}$ выражались поразному через основные характеристики модели (у нас было 4 случая), в этом месте различия исчезают и разностный оператор во всех случаях одинаков.

Предельный спектральный интервал также легко считается:

$$
\lim _{\rho \rightarrow \infty}\left[\frac{(N-1)(N+\alpha+\beta)}{\rho^{2}}, 0\right]=[-\widetilde{N}(\tilde{N}+\widetilde{T}), 0] .
$$

Обозначим через $I_{[a, b]}$ индикатор отрезка $[a, b]$.

Заметим, что справедливо тождество

$$
\widehat{P}=I_{[-\widetilde{N}(\widetilde{N}+\widetilde{T}), 0]}(\widehat{H})=I_{\left[\frac{-\widetilde{N}(\widetilde{N}+\widetilde{T})-A}{2 B},-\frac{A}{2 B}\right]}\left(\frac{\widehat{H}-A}{2 B}\right) .
$$

$(\widehat{H}-A) /(2 B)$ является оператором, в матрице которого над и под главной диагональю стоит $1 / 2$, а в остальных местах - нуль.

Для того чтобы явно вычислить $\widehat{P}$, сделаем преобразование Фурье $l_{2}(\mathbb{Z}) \rightarrow$ $L_{2}\left(S^{1}\right)$, где $S^{1}$ - единичная окружность в $\mathbb{C}$.

Фурье-образ оператора $(\widehat{H}-A) /(2 B)$ есть оператор умножения на $(z+\bar{z}) / 2=$ $\operatorname{Re} z$. Заметим, что его спектр непрерывен и заполняет отрезок $[-1,1]$. Отсюда можно заключить, что и спектр оператора $\widehat{H}$ непрерывен (это условие нам необходимо для применения теоремы VII.24 из [20]).

Далее, при подстановке $A$ и $B$ координата левого конца спектрального отрезка превращается в

$$
\frac{-\tilde{N}(\tilde{N}+\tilde{T})+(-\tilde{x}+\widetilde{S}+\tilde{N})(\tilde{t}+\tilde{N}-\tilde{x})+\tilde{X}(\widetilde{T}+\tilde{x}-\tilde{S}-\tilde{t})}{2 \sqrt{\tilde{x}(-\tilde{x}+\widetilde{S}+\tilde{N})(\tilde{t}+\tilde{N}-\tilde{x})(\tilde{x}+\widetilde{T}-\widetilde{S}-\tilde{t})}},
$$

а координата правого конца - в

$$
\frac{(-\tilde{x}+\widetilde{S}+\tilde{N})(\tilde{t}+\tilde{N}-\tilde{x})+\tilde{x}(\widetilde{T}+\tilde{x}-\tilde{S}-\tilde{t})}{2 \sqrt{\tilde{x}(-\tilde{x}+\widetilde{S}+\tilde{N})(\tilde{t}+\tilde{N}-\tilde{x})(\tilde{x}+\widetilde{T}-\widetilde{S}-\tilde{t})}} .
$$

Заметим, что последнее выражение больше единицы. Следовательно, фурьеобраз спектрального проектора $\widehat{P}$ сводится к оператору умножения на индикатор правой дуги единичной окружности, заключенной между углами - $\phi$ и $\phi$, где

$$
\phi=\arccos \frac{-\tilde{N}(\tilde{N}+\widetilde{T})+(-\tilde{x}+\widetilde{S}+\tilde{N})(\tilde{t}+\tilde{N}-\tilde{x})+\tilde{x}(\widetilde{T}+\tilde{x}-\widetilde{S}-\tilde{t})}{2 \sqrt{\tilde{x}(-\tilde{x}+\widetilde{S}+\tilde{N})(\tilde{t}+\tilde{N}-\tilde{x})(\tilde{x}+\widetilde{T}-\widetilde{S}-\tilde{t})}} .
$$


Сделав обратное преобразование Фурье, находим матрицу оператора $\widehat{P}$, совпадающую с искомым корреляционным ядром

$$
K(\hat{x}, \hat{y})=\frac{1}{2 \pi i} \oint_{e^{-i \phi}}^{e^{i \phi}} w^{\hat{y}-\hat{x}-1} d w=\frac{1}{2 \pi i} \oint_{e^{-i \phi}}^{e^{i \phi}} w^{\hat{x}-\hat{y}-1} d w
$$

(интегрирование происходит по правой дуге единичной окружности).

Последний интеграл явно считается и совпадает с дискретным синус-ядром:

$$
K(\hat{x}, \hat{y})=\frac{\sin (\phi(x-y))}{\pi(x-y)} .
$$

Заметим, что таким же методом мы могли бы найти предел ядра

$$
\breve{K}_{t}(x, y)=-\sum_{i \geqslant N} f_{i}^{t}(x) f_{i}^{t}(y)
$$

Этот предел равняется

$$
\breve{K}(\hat{x}, \hat{y})=\frac{1}{2 \pi i} \oint_{e^{-i \phi}}^{e^{i \phi}} w^{\hat{x}-\hat{y}-1} d w
$$

где интегрирование производится по левой дуге единичной окружности. В реализации в $L_{2}\left(S^{1}\right)$ мы получаем оператор умножения на индикатор левой дуги окружности.

3.3. Переход к динамическому ядру. Пусть теперь $s<t$. Имеем

$$
K(x, s ; y, t)=-\sum_{i \geqslant N}\left(\prod_{j=s}^{t-1} c_{i}^{j}\right) f_{i}^{s}(x) f_{i}^{t}(y) .
$$

Зафиксируем $s$ и $t$, и пусть 2 - оператор в $l_{2}(\mathbb{Z})$ с матрицей

$$
\mathcal{Q}(x, y)= \begin{cases}K(x, s ; y, t), & x \in \mathfrak{X}_{s}, y \in \mathfrak{X}_{t}, \\ 0 & \text { для остальных } x, y .\end{cases}
$$

Этот оператор можно представить в виде

$$
\mathcal{Q}=\mathscr{P}_{t} U_{t-1} U_{t-2} \cdots U_{s} .
$$

Здесь $\mathscr{P}_{t}$ - оператор, отвечающий рассмотренному в конце предыдущего раздела ядру $\breve{K}_{t}(x, y)$, т. е. оператор с матрицей

$$
\mathscr{P}_{t}(x, y)= \begin{cases}-\sum_{i \geqslant N} f_{i}^{t}(x) f_{i}^{t}(y), & x, y \in \mathfrak{X}_{t}, \\ 0 & \text { для остальных } x, y,\end{cases}
$$

а $U_{h}$ - оператор, отображающий каждую функцию $f_{i}^{h}(x)$ в $c_{i}^{h} f_{i}^{h+1}(x)$ (если в момент времени $h+1$ многочленов на 1 меньше, то функция, отвечающая многочлену старшей степени, переходит в нуль; это согласовано с тем, что соответствующий коэффициент $c_{i}^{h}$ обращается в нуль). На ортогональном дополнении к линейной оболочке функций $f_{i}^{h}$ положим $U_{h}$ тождественно равным нулю. 
В матричном виде этот оператор представляется так:

$$
U_{h}(x, y)= \begin{cases}\sum_{i \geqslant 0} c_{i}^{h} f_{i}^{h}(x) f_{i}^{h+1}(y), & x \in \mathfrak{X}_{h}, y \in \mathfrak{X}_{h+1}, \\ 0 & \text { для остальных } x, y .\end{cases}
$$

Выразив, как и в случае статического ядра, все параметры через характеристики предельного режима, получим семейство операторов, зависящее от параметра $\rho$. Теперь для нахождения предела $\mathcal{Q}_{\rho}$ при $\rho \rightarrow \infty$ перейдем к пределу отдельно в каждом из сомножителей и воспользуемся следующим соображением: все сомножители сильно сходятся на всюду плотном множестве финитных векторов, норма каждого из сомножителей не больше единицы (для операторов $U_{h}$ это следует из того, что $\left.0 \leqslant c_{i}^{j} \leqslant 1\right)$; следовательно, эти сомножители сильно сходятся на всем пространстве $l_{2}(\mathbb{Z})$. Операция умножения операторов непрерывна по совокупности переменных в сильной операторной топологии при условии равномерной ограниченности норм всех сомножителей. Это позволяет нам сделать заключение о сильной сходимости и для операторов $\mathcal{Q}_{\rho}$.

Заметим, что матрица $U_{h}$ нами уже была фактически вычислена при доказательстве детерминантности процесса:

$$
\begin{aligned}
& U_{h}(x, y)=v_{h, h+1}(x, y) \\
& =\sqrt{\frac{w_{h}(x)}{w_{h+1}(y)}}\left[\frac{S+N-1-x}{\sqrt{(h+N)(T+N-h-1)}} \delta_{x+1}^{y}+\frac{T-h-S+x}{\sqrt{(h+N)(T+N-h-1)}} \delta_{x}^{y}\right] \\
& =\delta_{x+1}^{y} \sqrt{\frac{(S+N-x-1)(x+1)}{(h+N)(T+N-h-1)}+} \delta_{x}^{y} \sqrt{\frac{(T-h-S+x)(h+N-x)}{(h+N)(T+N-h-1)}} \\
& \left(x \in \mathfrak{X}_{h}, y \in \mathfrak{X}_{h+1}\right) .
\end{aligned}
$$

Подставим сюда параметры нашего предельного перехода

$$
\begin{gathered}
S=\rho \widetilde{S}+o(\rho), \quad T=\rho \widetilde{T}+o(\rho), \quad N=\rho \tilde{N}+o(\rho), \\
t=\rho \tilde{t}+\hat{t}, \quad t=\rho \tilde{t}+\hat{s}, \quad x=\rho \tilde{x}+\hat{x}
\end{gathered}
$$

и получим оператор $U_{h, \rho}$. Учитывая, что $s \leqslant h<t$, устремляем $\rho$ к бесконечности. Получаем оператор с матрицей

$$
\widehat{U}(\hat{x}, \hat{y})=\delta_{\hat{x}+1}^{\hat{y}} \sqrt{\frac{(\widetilde{S}+\widetilde{N}-\tilde{x})(\tilde{x})}{(\tilde{t}+\widetilde{N})(\widetilde{T}+\widetilde{N}-\tilde{t})}}+\delta_{\hat{x}}^{\hat{y}} \sqrt{\frac{(\widetilde{T}-\tilde{t}-\widetilde{S}+\tilde{x})(\tilde{t}+\tilde{N}-\tilde{x})}{(\tilde{t}+\widetilde{N})(\widetilde{T}+\widetilde{N}-\tilde{t})}} .
$$

Сделаем преобразование Фурье и перемножим все операторы, в произведение которых распался предельный оператор $\widehat{\mathcal{Q}}$. Получим оператор умножения на произведение функции

$$
\left(\sqrt{\frac{(\widetilde{T}-\tilde{t}-\widetilde{S}+\tilde{x})(\tilde{t}+\widetilde{N}-\tilde{x})}{(\tilde{t}+\widetilde{N})(\widetilde{T}+\widetilde{N}-\tilde{t})}}+\frac{1}{z} \sqrt{\frac{(\widetilde{S}+\widetilde{N}-\tilde{x}) \tilde{x}}{(\tilde{t}+\widetilde{N})(\widetilde{T}+\widetilde{N}-\tilde{t})}}\right)^{\hat{t}-\hat{s}}
$$

и индикатора левой дуги окружности, заключенной между углами $\phi$ и $2 \pi-\phi$. 
Сделав обратное преобразование Фурье, получаем искомое корреляционное ядро:

$$
\begin{aligned}
& K(\hat{x}, \hat{s} ; \hat{y}, \hat{t}) \\
& =\frac{1}{2 \pi i} \oint_{e^{-i \phi}}^{e^{i \phi}}\left(\sqrt{\frac{(\widetilde{T}-\tilde{t}-\widetilde{S}+\tilde{x})(\tilde{t}+\widetilde{N}-\tilde{x})}{(\tilde{t}+\widetilde{N})(\widetilde{T}+\widetilde{N}-\tilde{t})}}+\frac{1}{z} \sqrt{\frac{(\widetilde{S}+\widetilde{N}-\tilde{x}) \tilde{x}}{(\tilde{t}+\widetilde{N})(\widetilde{T}+\widetilde{N}-\tilde{t})}}\right)^{\hat{t}-\hat{s}} z^{\hat{y}-\hat{x}-1} d z \\
& \stackrel{w=1 / z}{=} \frac{1}{2 \pi i} \oint_{e^{-i \phi}}^{e^{i \phi}}\left(\sqrt{\frac{(\widetilde{T}-\tilde{t}-\widetilde{S}+\tilde{x})(\tilde{t}+\widetilde{N}-\tilde{x})}{(\tilde{t}+\widetilde{N})(\widetilde{T}+\widetilde{N}-\tilde{t})}}+w \sqrt{\frac{(\widetilde{S}+\widetilde{N}-\tilde{x}) \tilde{x}}{(\tilde{t}+\widetilde{N})(\widetilde{T}+\widetilde{N}-\tilde{t})}}\right)^{\hat{t}-\hat{s}} w^{\hat{x}-\hat{y}-1} d w \\
& =\left(\sqrt{\frac{(\widetilde{T} \tilde{t}-\widetilde{S}+\tilde{x})(\tilde{t}+\tilde{N}-\tilde{x})}{(\tilde{t}+\widetilde{N})(\widetilde{T}+\widetilde{N}-\tilde{t})}}\right)^{\hat{t}-\hat{s}} \frac{1}{2 \pi i} \oint_{e^{-i \phi}}^{e^{i \phi}}(1+c w) w^{\hat{x}-\hat{y}-1} d w .
\end{aligned}
$$

Множитель перед интегралом можно опустить, так как он соответствует лишь сопряжению ядра, не меняющему корреляционных функций. В результате мы получили выражение из формулировки теоремы.

В случае $s>t$ рассуждения аналогичны, приведем их в сокращенном виде. Рассматриваем оператор $\mathscr{G}$ с матрицей

$$
\mathscr{G}(x, y)= \begin{cases}K(x, s ; y, t), & x \in \mathfrak{X}_{s}, y \in \mathfrak{X}_{t}, \\ 0 & \text { для остальных } x, y\end{cases}
$$

Его можно представить в виде

$$
\mathscr{G}=V_{s-1} \cdots V_{t+1} V_{t} \mathscr{R} .
$$

Здесь $V_{h}$ есть оператор, заданный на первых $N$ функциях $f_{i}^{h}$ формулой $V_{h}\left(f_{i}^{h}\right)=$ $f_{i}^{h+1} / c_{i}^{h}$. Как и раньше, мы затем продолжаем его на все пространство. Оператор $\mathscr{R}$ есть ортопроектор на первые $N$ функций $f_{i}^{t}$.

Выражая операторы через параметры предельного режима, получаем семейства операторов $\mathscr{G}_{\rho}, V_{h, \rho}$ и $\mathscr{R}_{\rho}$.

Из определения чисел $c_{i}^{h}$ мы снова можем заключить, что нормы операторов $V_{h, \rho}$ и $\mathscr{R}_{\rho}$ ограничены.

$\widehat{\mathscr{R}}$ - предел операторов $\mathscr{R}_{\rho}$ при $\rho \rightarrow \infty-$ нами уже подсчитан в предыдущем разделе. Он равен оператору ограничения на правую дугу окружности, заключенную между углами $-\phi$ и $\phi$.

Теперь остается воспользоваться следующей леммой:

Лемма 9. Пусть имеется два семейства операторов $A_{\rho}, B_{\rho}$ в гильбертовом пространстве. Пусть при $\rho \rightarrow \infty$ нормы всех операторов ограничены некоторой константой, $A_{\rho} \rightarrow A u B_{\rho} A_{\rho} \rightarrow P$ в смысле сильной операторной сходимости, $P$ - некоторый ортопроектор, $E$ - его образ. Предполагается, что подпространство $Е$ является инвариантным относительно оператора $A$ и ограничение последнего на это подпространство, $A_{E}$, обратимо на Е. Тогда на подпространстве $E$ операторь $B_{\rho}$ силъно сходятся $\kappa A_{E}^{-1}$. 
Доказательство. Пусть $x-$ произвольный вектор, лежащий в образе оператора $P$. Из условия следует, что найдется такой $y$, что $x=A y$. Тогда

$$
\begin{aligned}
B_{\rho} A_{\rho} y & \rightarrow y, \quad A_{\rho} y \rightarrow x, \\
B_{\rho} x=B_{\rho}\left(A_{\rho} y+\left(x-A_{\rho} y\right)\right) & =B_{\rho} A_{\rho} y+B_{\rho}\left(x-A_{\rho} y\right) \rightarrow y+0=y .
\end{aligned}
$$

Применим доказанную лемму к $A_{\rho}=U_{h+1, \rho}$ и $B_{\rho}=V_{h, \rho}$. Произведение $B_{\rho} A_{\rho}$ совпадает с оператором, матрица которого задается ядром, рассмотренным в статическом случае, а потому сходится к проектору $\widehat{\mathscr{R}}$, а операторы $A_{\rho}$, как было показано выше, сходятся к оператору $\widehat{U}$, который в реализации в $L_{2}\left(S^{1}\right)$ является оператором умножения на функцию

$$
\sqrt{\frac{(\widetilde{T}-\tilde{t}-\widetilde{S}+\tilde{x})(\tilde{t}+\widetilde{N}-\tilde{x})}{(\tilde{t}+\widetilde{N})(\widetilde{T}+\widetilde{N}-\tilde{t})}}+\frac{1}{z} \sqrt{\frac{(\widetilde{S}+\widetilde{N}-\tilde{x}) \tilde{x}}{(\tilde{t}+\widetilde{N})(\widetilde{T}+\widetilde{N}-\tilde{t})}} .
$$

Эта функция при ограничении на единичную окружность гарантированно отлична от нуля и обратима при $z \neq-1$, но при некоторых значениях параметров предельного режима обращается в нуль при $z=-1$. Однако предельный проектор $\widehat{\mathscr{R}}$ является оператором умножения на индикатор некоторой дуги, не содержащей точки $z=-1$. Следовательно, при ограничении на образ проектора $\widehat{\mathscr{R}}$ оператор $\widehat{U}$ обратим, и мы можем воспользоваться леммой.

Получаем, что (в реализации в пространстве $\left.L_{2}\left(S^{1}\right)\right)$ предел операторов $\mathscr{G}_{\rho}$ умножает векторы из образа предельного проектора $\widehat{\mathscr{R}}$ на некоторую функцию. Эта функция является обратной к той, на которую умножает оператор $\widehat{U}$. С другой стороны, на векторах из ортогонального дополнения к образу проектора $\mathscr{R}_{\rho} \rightarrow 0$, а следовательно, так как нормы операторов $V_{h, \rho}$ ограничены, и $\mathscr{G}_{\rho} \rightarrow 0$.

Дальнейшие рассуждения дословно повторяют случай $s<t$. Теорема полностью доказана.

\section{§4. Анализ полученных результатов}

4.1. Замороженные области. Рассмотрим подробнее полученную одномерную корреляционную функцию, называемую также функцией плотности.

Уже было отмечено, что все точки, через которые могут проходить наборы непересекающихся путей, образуют шестиугольник, у которого одна пара сторон параллельна одной оси, вторая - другой, а третья пара наклонена под углом $45^{\circ}$.

В работе [8] рассматривались замощения шестиугольника ромбами, которые также могут быть интерпретированы в терминах непересекающихся путей. Было доказано существование «замороженных областей» (arctic region), в которых предельное замощение перестает быть случайным и состоит из ромбов одного типа (точную формулировку см. в теореме 1.1 в [8]). Ниже вкратце объясняется, что наши результаты согласованы с этой теоремой, и форму замороженной области можно определить с помощью нашего динамического корреляционного ядра как область значений параметров предельного режима, при которых локальные флуктуации исчезают и предельный точечный процесс становится тривиальным (либо с вероятностью 1 все точки решетки принадлежат случайной 
конфигурации, либо, напротив, с вероятностью 1 ни одна точка не принадлежит случайной конфигурации).

Зафиксируем в нашей модели параметры шестиугольника $\widetilde{N}, \widetilde{T}, \widetilde{S}$ и изучим зависимость функции плотности от точки $(\tilde{t}, \tilde{x})$.

Предложение 10. Распределение конфигуращий в нашей модели нетривиально только внутри эллипса, касающегося сторон шестиугольника, задаваемого параметрами $\widetilde{N}, \widetilde{T}, \widetilde{S}$, а вне него функиия плотности равна либо 1 , либо 0 .

Доказательство. Подсчитаем $K(\hat{x}, \hat{t} ; \hat{x}, \hat{t})$ - плотность распределения точек, через которые проходят пути. Имеем

$$
K=\frac{1}{2 \pi i} \oint_{-\phi}^{\phi} \frac{d w}{w}=\frac{\phi}{\pi} .
$$

При некоторых значениях параметров $\phi=0$ или $\phi=\pi$; это и означает, что плотность равняется нулю или единице соответственно. В наших терминах это эквивалентно тому, что в определяющем угол $\phi$ соотношении $\phi=\arccos D$ параметр $D$ по модулю не меньше единицы. Это можно записать в виде $D^{2} \geqslant 1$. После раскрытия скобок и упрощений последнее неравенство преобразуется к виду

$$
\begin{aligned}
\widetilde{T}^{2} \tilde{x}^{2} & +(\widetilde{S}+\widetilde{N})^{2} \tilde{t}^{2}+2 \tilde{x} \tilde{t}(\widetilde{N} \widetilde{T}-\widetilde{S} \widetilde{T}-2 \widetilde{S} \widetilde{N}) \\
& +2 \tilde{t}\left(\widetilde{S} \widetilde{N}^{2}-\widetilde{N} \widetilde{T} \widetilde{S}-\widetilde{N}^{2} \widetilde{T}+\widetilde{S}^{2} \widetilde{N}\right)+2 \tilde{x}\left(\widetilde{N} \widetilde{T} \widetilde{S}-\widetilde{N} \widetilde{T}^{2}\right)+\widetilde{N}(\widetilde{T}-\widetilde{S})^{2} \leqslant 0 .
\end{aligned}
$$

Это неравенство задает внутренность некоторого эллипса, и легко убедиться, что последний касается границ изучаемого шестиугольника, задаваемого параметрами $\widetilde{N}, \widetilde{T}, \widetilde{S}$.

В работе [8] замороженная область оказывалась точно такой же - внешностью эллипса, касающегося сторон шестиугольника.

4.2. Связь с моделью Окунькова-Решетихина. Установим связь полученного нами предельного динамического корреляционного ядра с ядром, полученным в работе [19]. Для изучаемого там точечного процесса ядро оказывалось равным

$$
K_{\mathrm{OR}}(x, s ; y, t)=\frac{1}{2 \pi i} \oint_{\bar{z}}^{z}(1-w)^{t-s} w^{x-y+(s-t) / 2-1} d w,
$$

где $z$ - некоторое комплексное число, по модулю меньшее 1, с положительной вещественной частью, зависящее от параметров модели, а интегрирование производится по правой дуге окружности при $t \geqslant s$ и по левой в противном случае.

Предложение 11. Можно выбрать линейное преобразование решетки $\mathbb{Z}^{2}$ так, что прочессы, задаваемые полученным нами корреляиионным ядром и ядром из [19], связаны инволюиией частицы-дырки, т.е. случайные конфигурации, описываемые одним ядром, являются дополнительными к случайным конфигураииям, описываемым вторым.

Доказательство. Заметим, что в изучаемой нами модели подбором пяти параметров можно добиться произвольных значений параметров ядра $c, \phi, 0<$ $c \leqslant 1$ и $0 \leqslant \phi \leqslant \pi$. 
Перейдем к модели из [19]. В интеграле, выражающем корреляционное ядро, можно сделать замену переменных, сводящую контур интегрирования к дуге единичной окружности (сразу можно заметить, что возникающий внеинтегральный множитель можно опустить, так как он задает некоторое сопряжение ядра, которое не меняет корреляционных функций). Кроме того, сделаем линейное преобразование решетки

$$
s^{\prime}-t^{\prime}=s-t, \quad x^{\prime}-y^{\prime}=x-y+(s-t) / 2 .
$$

На самом деле именно при этом преобразовании решетка, рассматриваемая Окуньковым и Решетихиным, переходит в $\mathbb{Z}^{2}$. В результате ядро приходит к виду

$$
K\left(x^{\prime}, s^{\prime} ; y^{\prime}, t^{\prime}\right)=\frac{1}{2 \pi i} \oint_{-\phi}^{\phi}(1-c w)^{t^{\prime}-s^{\prime}} w^{x^{\prime}-y^{\prime}-1} d w
$$

(интеграл берется по единичной окружности, выбор дуги интегрирования осуществляется по тем же правилам, что и раньше, $0<c \leqslant 1)$. При $s \neq t$ сделаем в полученном интеграле замену переменных $z=-w$ :

$$
K=\frac{(-1) \cdot(-1)^{x-y}}{2 \pi i} \oint_{-\phi}^{\phi}(1+c z)^{t^{\prime}-s^{\prime}} z^{x^{\prime}-y^{\prime}-1} d z .
$$

Здесь интегрирование уже происходит по правой стороне окружности при $t<s$ и по левой в противном случае. Множитель $(-1)^{x-y}$ снова можно опустить. Это ядро отличается от ядра из нашей модели лишь знаком при $s \neq t$, а при $s=t$ - только дугой интегрирования. Для получения нужной нам дуги интегрирования остается вычесть интеграл из вычета подынтегральной функции в точке 0 , т. е. из 1 при $x=y$ и из 0 при $x \neq y$. Таким образом, ядра двух полученных процессов связаны соотношением $K_{1}=\delta_{x=y, s=t}-K_{2}$. Но именно так ядро преобразуется при инволюции частицы-дырки (например, это доказано в приложении А.3 в [3]).

Заметим, что если в рассматриваемой нами модели оказалось, что в интеграле $c>1$, то можно сделать замену переменных $w=1 / z$; при этом константа $c$ заменяется на $1 / c$, но еще меняется показатель степени при $z$, а именно $x-y-1$ заменяется на $x-y+(s-t)-1$. Однако такое преобразование есть всего лишь еще одна линейная замена координат на решетке; следовательно, и в этом случае предложение доказано.

Полученный результат можно было предвидеть, исходя из родственности моделей, - в [19] рассматривался ансамбль трехмерных диаграмм Юнга без ограничений на размер, а в одной из интерпретаций нашей модели появляются трехмерные диаграммы Юнга, но с некоторыми ограничениями, - а также из гипотетической универсальности полученного ядра, заявленной в [19]. Одним из подтверждений универсальности являлось получение такого же ядра в [2] в «bulk scaling limit» цилиндрических разбиений. Наш результат можно рассматривать как еще одно подтверждение гипотезы универсальности.

\section{§5. Приложение. Различные интерпретации рассмотренной модели}

У изученной нами модели имеется множество различных комбинаторных интерпретаций. Покажем, как они получаются.

Рассмотрим набор путей, изучаемый на протяжении всей работы. 


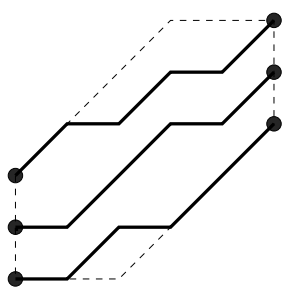

Рис. 2. Набор из 3 непересекающихся путей

Наклоняя под углом в $30^{\circ}$ вверх звенья ломаных, идущие под углом $45^{\circ}$, и заменяя горизонтальные наклоненными на $30^{\circ}$ вниз, мы получаем следующую картинку, которую можно интерпретировать как набор путей на поверхности трехмерной диаграммы Юнга:

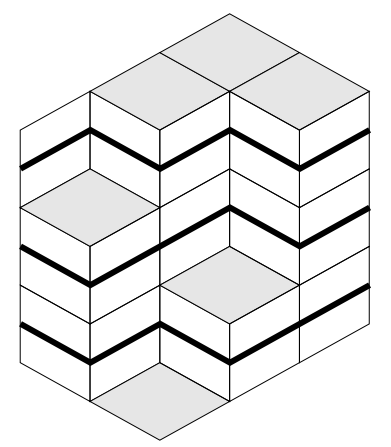

Рис. 3. Непересекающиеся пути на трехмерной диаграмме Юнга

Последнее изображение можно рассматривать и как разбиение шестиугольника на ромбики трех разных типов.

\section{ЛитеРАТУРА}

[1] J. Baik, T. Kriecherbauer, K. T.-R. McLaughlin, P. D. Miller, Discrete Orthogonal Polynomials: Asymptotics and Applications, Annals of Math. Studies, vol. 164, Princeton University Press, 2007; http://arxiv.org/abs/math/0310278.

[2] A. Borodin, Periodic Schur process and cylindric partitions, Duke Math. J., 140:3 (2007), 391-468; http://arxiv.org/abs/math/0601019.

[3] A. Borodin, A. Okounkov, G. Olshanski, Asymptotics of Plancherel measures for symmetric groups, J. Amer. Math. Soc., 13:3 (2000), 481-515; http://arxiv.org/abs/ math/9905032.

[4] A. Borodin, G. Olshanski, Markov processes on partitions, Probab. Theory Related Fields, 135:1 (2006), 84-152; http://arxiv.org/abs/math-ph/0409075.

[5] A. Borodin, G. Olshanski, Asymptotics of Plancherel-type random partitions, J. Algebra, 313:1 (2007), 40-60; http://arxiv.org/abs/math/0610240.

[6] Г. Бейтман, А. Эрдейи, Высшие трансцендентные функиии, т. 1, Физматлит, М., 1973.

[7] H. Cohn, R. Kenyon, J. Propp, A variational principle for domino tilings, J. Amer. Math. Soc., 14:2 (2001), 297-346; http://arxiv.org/abs/math/0008220.

[8] H. Cohn, M. Larsen, J. Propp, The shape of a typical boxed plane partition, New York J. Math. (electronic), 4 (1998), 137-165; http://arxiv.org/abs/math/9801059.

[9] B. Eynard, M. L. Mehta, Matrices coupled in a chain. I. Eigenvalue correlations, J. Phys. A: Math. Gen., 31:19 (1998), 4449-4456. 
[10] I. Gessel, G. Viennot, Binomial determinants, paths, and hook length formulae, Adv. Math., 58:3 (1985), 300-321.

[11] K. Johansson, Non-intersecting, simple, symmetric random walks and the extended Hahn kernel, Ann. Inst. Fourier (Grenoble), 55:6 (2005), 2129-2145; http:// arxiv.org/abs/math.PR/0409013.

[12] K. Johansson, E. Nordenstam, Eigenvalues of GUE minors, Electron. J. Probab., 11 (2006), no. 50, 1342-1371; http://arxiv.org/abs/math/0606760.

[13] R. Kenyon, Height fluctuations in the honeycomb dimer model; http://arxiv.org/ abs/math-ph/0405052.

[14] R. Kenyon, A. Okounkov, S. Sheffield, Dimers and Amoebae, Ann. of Math., 163:3 (2006), 1019-1056; http://arxiv.org/abs/math-ph/0311005.

[15] R. Koekoek, R. F. Swarttouw, The Askey-scheme of hypergeometric orthogonal polynomials and its q-analogue, http://aw.twi.tudelft.nl/ koekoek/reports.html.

[16] C. Krattenthaler, Advanced determinant calculus, Sém. Lothar. Combin. (electronic), 42 ("The Andrews Festschrift") (1999), Article B42q.

[17] M. L. Mehta, Random Matrices, 2nd ed., Academic Press, Boston, MA, 1991.

[18] A. Okounkov, Symmetric functions and random partitions. Symmetric functions 2001: surveys of developments and perspectives, in: NATO Sci. Ser. II Math. Phys. Chem., vol. 74, Kluwer Acad. Publ., Dordrecht, 2002, 223-252; http://arxiv.org/ $\mathrm{abs} / \mathrm{math} . \mathrm{CO} / 0309074$.

[19] A. Okounkov, N. Reshetikhin, Correlation functions of Schur process with application to local geometry of a random 3-dimensional Young diagram, J. Amer. Math. Soc., 16:3 (2003), 581-603; http://arxiv.org/abs/math.c0/0107056.

[20] М. Рид, Б. Саймон, Методы современной математической физики, m. 1, Функииональный анализ, Мир, М., 1977.

[21] S. Sheffield, Random Surfaces, Asterisque, no. 304, 2005; http://arxiv.org/abs/ math/0304049.

Московский государственный университет, механико-математический факультет

Поступило в редакцию e-mail: vadicgor@rol.ru 